\title{
Application of File Splitter in the Rapid Saving and Fetching Blob Data
}

\author{
Qu Baojun, Zhang YuPing \\ School of Mechanical Engineering \\ Shandong University of Technology \\ Zibo, China \\ qbj22@sina.com
}

\author{
$\mathrm{Hu}$ Lvbing \\ Jiujiang 707 Institute of Precision Mechatronics \\ SCI\&TECH Co. Ltd \\ China Shipbuilding Industry Company Limited \\ JiuJiang, China
}

\begin{abstract}
The Blob saving ways in SQL Server and disk saving theory of files in operation systems is analyzed in this paper. A method of splitting files in the Blob saving is given, which has been proved to be available to improve the efficiency of saving multimedia data in PDM system by experiments.
\end{abstract}

Keywords-SQL Server, BLOB, B-Tree, Split

\section{INTRODUCTION}

Now in PDM system, when people carry on the work of CAD and CAM, they need to deal with the multimedia data of grapf,image,voice,video and so on which make the hit of multimedia materials in PDM electronical data office more and more frequently. So the speed of storage and hit to these data has become a key question. Many databases in existence provide support to access of the larger field,for example, ORACLE, SQL Server and so on.In database system,these larger fields are called BLOB ( binary system large data).The access method to the data is different with the change of the programming language and the database.Therefore, the access to BLOB data type especially the research about how to access it fast has a very important meaning. The priority research in this paper is to get performance optimization by cutting up BLOB data in SQL Server.

\section{THE BLOB Storage Mode IN SQL SERVER}

\section{A. The Summary Of BLOB Data Type}

BLOB is indefinite binary system or categorical data which is tremendous. Usually it is file (.txt 、.doc)and picture (.jpeg、 .gif、.bmp), and it can be stored in databases. In SQL Server, BLOB can be text、ntext or image date type.

Text Non-Unicode data whose length is uncertainty is stored in server code page and the maximum length is $2^{\wedge} 31$ 1(2 147482647$)$ characters.

Ntext Unicode data whose length is uncertainty has the

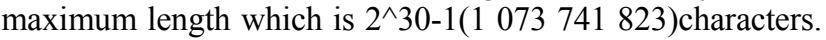
The storage in bytes of the field number is twice than the number of characters being entered. In SQL-92,ntext is a contraction of national text.

Image Binary data whose length is uncertainty. ranges from 0 to $2^{\wedge} 31-1(2147482647)$ bytes.

SQL Server takes the same way to handle these datatype. The way that SQL Server store image data is the same with it store text or ntext date type in the same conditions. Therefore, text、ntext and image date are usually called as text data.

Each row of text、ntext and image can contain not more than 2GB BLOB data in the table.The BLOB data is stored in the group of $8 \mathrm{~KB}$ data page, which is separated form other data page stored in the same table.The data page is arrayed in the structure of B-tree.

\section{B. BLOB's Storage Mode}

BLOB's storage is divided into out-of-row BLOB and inrow BLOB in SQL Server 2000.

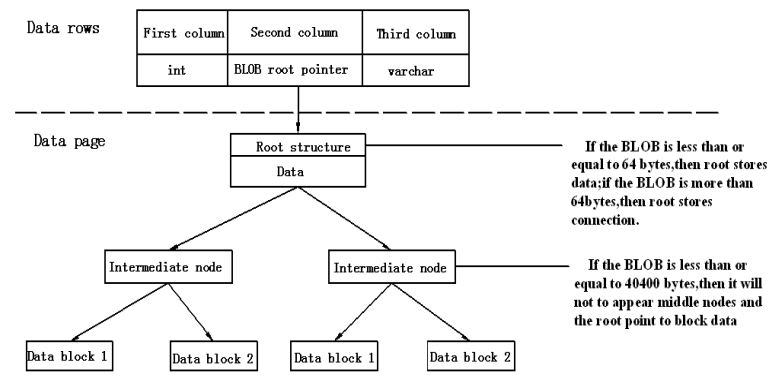

Figure 1. The storage data structure of BLOB data in SQL Server

\section{(1) Out-Of-Row BLOB}

When text in row isn't activated, BLOB is stored out of the row and only BLOB root pointer is stored in text ntext or image row. BLOB root pointer points to the root of B-tree structure which actual stores BLOB data. BLOB data is stored in root structure when its dimension is less than or equal to 64 bytes. The storage of root structure mapped to the connection of the data's path while the data is stored in Btree's leaf node when BLOB data's dimension is more than 64 bytes.

\section{(2) Inrow BLOB}

When text in row is activated, if BLOB data's dimension is less than or equal to limitation within row and the space is big enough to contain BLOB data then SQL Server will store the data in the data row.In row, Text in row is limited to apply to the fist BLOB row. Therefore, if the limitation within row is 256 bytes, and then the BLOB column in each row can store the data not more than 256 
bytes as long as the total number of the byte in the line is not more than 8060 bytes.

Though BLOB string is too long compared to data line, and SQL Server can access data more quickly when it is stored in single page. The path that SQL Server hit BLOB data is cut down on account that root structure is stored in data line.

\section{SPLIT AND ACCESS BLOB DATA}

\section{A. Principle Analysis}

B-tree which is usually applied to database and file system is a kind of tree data structure. B-tree can make the material stay organized.Besides it has the even movement to input and delete the time of logarithm process. The element in B-tree is inserted by using bottom-up method which is different from the most binary tree which is inserted by using top-down method

Because BLOB data is stored in disk in form of B-tree structure in SQL Serve so when we read or write a BLOB data each time, we should start with root node and find the needed block data following subtree .Finally a integrated file will be formed and stored into database. Supposing that the B-tree's number of nodes is $\mathrm{n}$ and the height is $\mathrm{H}$, after visiting the node whose depth is $\mathrm{h}(1<\mathrm{h}<\mathrm{H})$, if it wants to visit the node whose depth is $\mathrm{h}+1$,it is inevitable to visit the node whose depth ranges from 1 to and is bound to cause visiting the same block data repeatedly. So in order to visit BLOB data in disk, the disk access time will be $\mathrm{O}\left(\mathrm{n}^{*} \mathrm{~h}\right)$.

If a BLOB data is divided into $n$ equally sized files which are stored in sequential organization in adjacent record. Suppose the time for hitting each file is $0(1)$,after hitting the $\mathrm{i}$-th record and then hitting the i+1-th record directly, it doesn't need to go through the i-th record. So the time for hitting the total disk is $0(\mathrm{n})$. Compare global storage with dividual storage. When $\mathrm{n}$ is comparatively large and $\mathrm{h}$ is , then $\mathrm{O}(\mathrm{n})$ is for less than $\mathrm{O}(\mathrm{n} * \mathrm{~h})$. therefore, theoretically, it's obvious to improve storage efficiency by dividing BLOB data and storing into database separately.

In a computer, all files is stored in storage media in binary form and is handled in binary form ,too. So we can divide the big data file into divers data equal in length and then write a new file separately. Combination is connecting all divided files orderly.

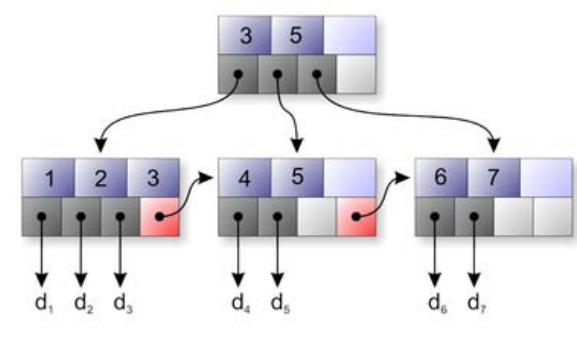

Figure 2. A simple B-tree

From figure 2, we can see a simple B-tree structure.

\section{B. Storage Experiment}

PDM application can use a variety of database access technology to access the SQL Server database. For quick and easy access to BLOB data, the experiment using ADO technology, because $\mathrm{ADO}$ is easy to use, fast, memory consumption, compared to ODBC, DAO, OLE DB and ADO database access technology.

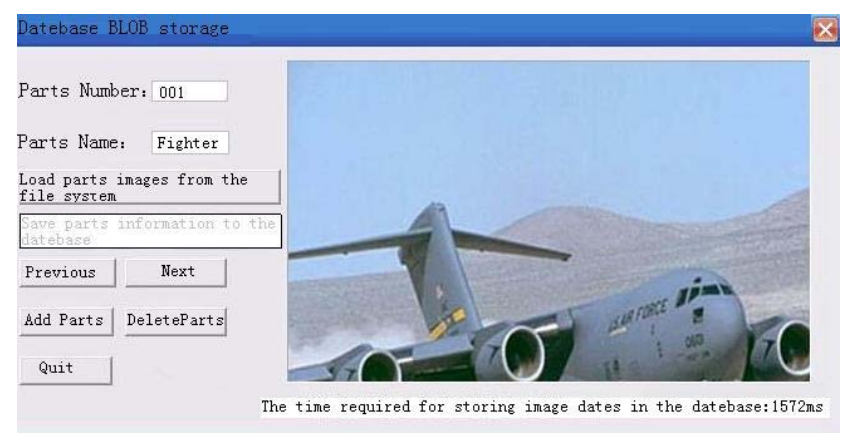

Figure 3. BLOB Datebase Storage Interface

Program interface shown in figure 3, the experiment environment is: high-speed local area network, the server is Intel PentiumIV, CPU1.7GHz,EMS memory $256 \mathrm{MB}$, the operating system is Windows 2000 Professional SP6; the client is Intel Pentium III processor, CPU is $583 \mathrm{MHz}, \mathrm{RAM}$ is $192 \mathrm{MB}$, the operating system is Windows XP Pro, 18 aircraft image files are stored in the client machine and it use the design process to a server database to store pictures.

When you store a single image, the storage time is not stable, so the random sampling of 18 aircraft ranging in size picture data, respectively, are stored in SQL Server database. In order to eliminate uncertainty in the operation, each image is stored five times. Experimental data are shown in Table I:

TABLE I. THE STORAGE TIME OF UNDIVIDED BLOB DATA

\begin{tabular}{cccccccccc}
\hline Number & 1 & 2 & 3 & 4 & 5 & 6 & 7 & 8 & 9 \\
\hline Byte(M) & 2.25 & 3.08 & 4.08 & 5.06 & 6.94 & 8.58 & 9.44 & 10.3 & 11.3 \\
\hline & 1422 & 1032 & 2250 & 2188 & 2828 & 5657 & 5079 & 8172 & 6594 \\
$\begin{array}{c}\text { Storage time } \\
\text { (ms) }\end{array}$ & $\mathbf{4 8 9 4}$ & 1687 & $\mathbf{2 6 5 6}$ & $\mathbf{3 1 0 9}$ & $\mathbf{3 5 0 0}$ & 4547 & 4687 & 5625 & 11578 \\
& 1109 & 1531 & 2422 & 2328 & 3156 & 3093 & 4922 & 4640 & 5140 \\
& 719 & 1203 & 1891 & 2203 & 3485 & 4953 & 4563 & 4875 & 11734 \\
\hline $\begin{array}{c}\text { Average time } \\
\text { (ms) }\end{array}$ & 1065.6 & 1378 & 2131.2 & 2587.4 & 3225.2 & 4334.4 & 4519 & 5756.2 & $\mathbf{8 8 7 5}$ \\
\hline Number & 10 & 11 & 12 & 13 & 14 & 15 & 16 & 17 & 18 \\
\hline Byte (M) & 13.4 & 14.4 & 16.8 & 18.0 & 19.3 & 20.2 & 21.9 & 23.3 & 34.3 \\
\hline & 6937 & $\mathbf{8 2 5 0}$ & 12594 & 15172 & 14828 & 13219 & 21907 & 39016 & 70781 \\
& 12719 & 14172 & 17860 & 21344 & 26093 & 24078 & 27250 & 48828 & 56265 \\
$\begin{array}{c}\text { Storage time } \\
\text { (ms) }\end{array}$ & 11594 & 14782 & 18687 & 24359 & 30547 & 15531 & 26438 & 46921 & 74266 \\
& $\mathbf{8 5 4 6}$ & 7188 & 11875 & 13437 & 23172 & 28563 & 19813 & 29969 & 76234 \\
& 13969 & 7454 & 18531 & 14016 & 15188 & 23109 & 18922 & 20938 & 52141 \\
\hline $\begin{array}{c}\text { Average time } \\
\text { (ms) }\end{array}$ & 10753 & 10369 & 15909 & 17665.6 & 21966 & 20900 & 22866 & 37134 & 65937 \\
\hline
\end{tabular}

Experimental results show that, for each image file, when we store them at different times in the database, sometimes the differences of time-consuming are very large. But when we calculate the average time, we can find that both of the 
general trends are consistent: the storage time increase with the picture file size in bytes' growth.

Compared with the analysis of large databases bytes of data storage time, a numerical analysis software Matlab to draw a large size in bytes of data - storage time curve shown in Figure 4 。

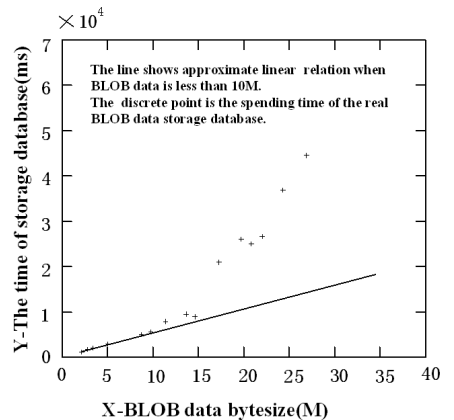

Figure4. The storage time of undivided BLOB data

We can see from Figure 4, when the BLOB data's size is less than 10 megabytes, the storage time increased linearly, and when the BLOB date's size is larger than 10 megabytes bytes, approximate growth rate increased exponentially. For example, it is easy to see when the BLOB date is 7 megabytes, the time stored in the database is about $3300 \mathrm{~ms}$, and if we store 5 same size of the BLOB date, the total time is just 17 seconds. When the BLOB data size increased to 35 megabytes, the storage time is 67 seconds. It can be seen from the above figures, split the data before storing them into the database can save many time.

Therefore, according to the experimental results curves, for the size of more than $15 \mathrm{M}$ bytes of images, graphics and other large data files, before they are stored into databases, you should first split them into a number of files in $5 \sim 10 \mathrm{M}$ ones, and then put these small files into the database's adjacent records one by one in order to increase storage efficiency. When users need to use these data, then they can merge these small files, restore them to the original documents.

For example, for the size of $34.3 \mathrm{M}$ bytes of image files, before you store them into the database,you can divide it into 7 files. The six files is $5 \mathrm{M}$, and the last one is $4.3 \mathrm{M}$,then store them in the database's adjacent records. The storage time is as follows. the measured storage time follows table II below.

TABLE II. THE STORAGE TIME OF DIVIDED BLOB DATA

\begin{tabular}{|c|c|c|c|c|c|c|c|c|c|}
\hline Number & 1 & 2 & 3 & 4 & 5 & 6 & 7 & 8 & 9 \\
\hline Byte (M) & 2.25 & 3.08 & 4.08 & 5.06 & 6.94 & 8.58 & 9.44 & 10.3 & 11.3 \\
\hline \multirow{5}{*}{$\begin{array}{l}\text { Storage time } \\
\text { (ms) }\end{array}$} & 1422 & 1032 & 2250 & 2188 & 2828 & 5657 & 5079 & 8172 & 6594 \\
\hline & 1594 & 1687 & 2656 & 3109 & 3500 & 4547 & 4687 & 5625 & 11578 \\
\hline & 484 & 1438 & 1437 & 3109 & 3157 & 3422 & 3344 & 5469 & 9328 \\
\hline & 1109 & 1531 & 2422 & 2328 & 3156 & 3093 & 4922 & 4640 & 5140 \\
\hline & 719 & 1203 & 1891 & 2203 & 3485 & 4953 & 4563 & 4875 & 11734 \\
\hline $\begin{array}{c}\text { Average time } \\
(\mathrm{ms})\end{array}$ & 1065.6 & 1378 & 2131.2 & 2587.4 & 3225.2 & 4334.4 & 4519 & 5756.2 & 8875 \\
\hline Number & 10 & 11 & 12 & 13 & 14 & 15 & 16 & 17 & 18 \\
\hline Byte (M) & 13.4 & 14.4 & 16.8 & 18.0 & 19.3 & 20.2 & 21.9 & 23.3 & 34.3 \\
\hline
\end{tabular}

\begin{tabular}{cccccccccc}
\hline & 6936 & 8057 & 7964 & 8272 & 8628 & 9247 & 9731 & 17367 & 24961 \\
Storage time & 13772 & 12682 & 13837 & 14653 & 15347 & 17935 & 18258 & 20471 & 29578 \\
(ms) & 14854 & 14974 & 15936 & 15973 & 16739 & 18259 & 19683 & 21963 & 30603 \\
& 11963 & 12827 & 13148 & 12684 & 13473 & 11364 & 12349 & 15256 & 27843 \\
& 8327 & 9671 & 10759 & 9854 & 10537 & 12982 & 15267 & 12862 & 19374 \\
\hline Average time (ms) & 11170.4 & 11642.2 & 12328.8 & 12287.2 & 12944.8 & 13957.4 & 15057.6 & 17584 & 26472 \\
\hline
\end{tabular}

The date from table 2 can be seen, after split the image file, the average time which storing the date into the database is about $26 \mathrm{~s}$, compared with the storage time of undivided, is significantly reduced.

To analysis the storage time after split the large bytes of date; we use numerical analysis software Matlab to draw upon a large partition size in bytes of bytes of data - storage time curve shown in Figure 5.

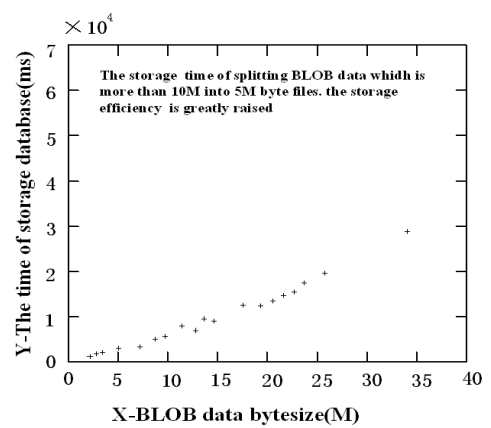

Figure 5. The storage time of divided BLOB data

Experiments date from Figure 5 show that after you split the BLOB data more than $10 \mathrm{M}$, and then store them into the datebase's adjacent records, the storage time will be greatly reduced, and significantly improve the efficiency of data access .

\section{SUMMARIES}

We consider the requirements of the storage of database and handling large BLOB data slowness and access to shared resources of PDM, we divide the image files into several small pieces in the PDM system and store separately. We can see from the results, the use of file segmentation approach can significantly improve the BLOB data storage time and greatly improve the efficiency of data access. This method has great practical significance

\section{REFERENCES}

[1] Microsoft, the. Microsoft SQL Server 2000 Resource Kit [M]. Yun Zhou studio translation. Beijing: Mechanical Industry Press, 2002.

[2] Zhang li.Visual C++ Advanced Programming [M]. Beijing: Posts and Telecom Press, 2002.

[3] Yuan yi and other authors.Visual C++ Practice and improvement database development and engineering application of article [M]. Beijing: China Railway Press, 2006.

[4] Seeking technology.Visual C++6.0 Database development technology and engineering practice $[\mathrm{M}]$. Beijing: People's Posts and Telecommunications Press, 2004.

[5] Zhun chunhua,Zhang yubiao,Lu xinchun.BLOB Data type access method and its application. Computer Applications and Software [J], 2002,19 (10): $52-54$ 